\title{
$B E$ Is Not the Unique Homomorphism That Makes the Partee Triangle Commute
}

\author{
Junri Shimada \\ Tokyo Keizai Univeristy, Tokyo, Japan \\ Meiji Gakuin University, Tokyo, Japan \\ Keio University, Tokyo, Japan \\ junrishimada@gmail.com
}

\begin{abstract}
Partee (1986) claimed without proof that the function $\mathrm{BE}$ is the only homomorphism that makes the Partee triangle commute. This paper shows that this claim is incorrect unless "homomorphism" is understood as "complete homomorphism." It also shows that BE and $A$ are the inverses of each other on certain natural assumptions.
\end{abstract}

\section{Introduction}

In a famous and influential paper, Partee (1986) discussed type-shifting operators for NP interpretations, including lift, ident and BE:

$$
\begin{aligned}
\text { lift } & =\lambda x \lambda P . P(x), \\
\text { ident } & =\lambda x \lambda y \cdot[y=x], \\
\mathrm{BE} & =\lambda \mathscr{P} \lambda x . \mathscr{P}(\lambda y \cdot[y=x]) .
\end{aligned}
$$

She pointed out that these operators satisfy the equality $\mathrm{BE} \circ$ lift $=$ ident, so the following diagram, now often referred to as the Partee triangle, commutes.

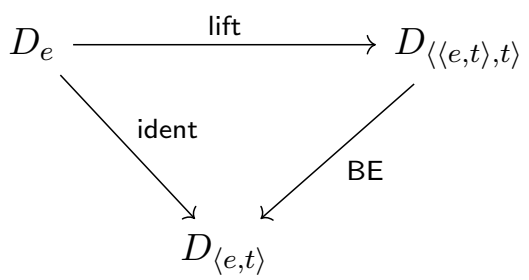

Diagram 1: The Partee triangle

Partee declared that BE is "natural" because of the following two "facts."

Fact 1. BE is a homomorphism from $\langle\langle e, t\rangle, t\rangle$ to $\langle e, t\rangle$ viewed as Boolean structures, i.e.,

$$
\begin{aligned}
\operatorname{BE}\left(\mathscr{P}_{1} \sqcap \mathscr{P}_{2}\right) & =\operatorname{BE}\left(\mathscr{P}_{1}\right) \sqcap \operatorname{BE}\left(\mathscr{P}_{2}\right), \\
\operatorname{BE}\left(\mathscr{P}_{1} \sqcup \mathscr{P}_{2}\right) & =\operatorname{BE}\left(\mathscr{P}_{1}\right) \sqcup \mathrm{BE}\left(\mathscr{P}_{2}\right), \\
\operatorname{BE}\left(\neg \mathscr{P}_{1}\right) & =\neg \operatorname{BE}\left(\mathscr{P}_{1}\right) .
\end{aligned}
$$

Fact 2. BE is the unique homomorphism that makes the diagram commute.

While Fact 1 is immediate, Fact 2 is not obvious. Partee (1986) nevertheless did not give a proof of Fact 2, but only a note saying, "Thanks to Johan van Benthem for the fact, which he knows how to prove but I don't." Meanwhile, van Benthem (1986) referred to Partee's work and stated Fact 2 on p. 68, but gave no proof either. Despite this quite obscure exposition, because of the classic status of Partee's and van Benthem's work, I suspect that many linguists take Fact 2 for granted while unable to explain it. Not only is this unfortunate, but it is actually as expected, because Fact 2 turns out to be not quite correct unless "homomorphism" is read as "complete homomorphism." The main purpose of this paper is to rectify this detrimental situation.

Van Benthem (1986) took the domain of entities to be finite, writing, "Our general feeling is that natural language requires the use of finite models only" (p. 7). Fact 2 is indeed correct on this assumption. However, natural language has predicates like natural number whose extensions are obviously infinite. Also, if we take the domain of portions of matter in the sense of Link (1983) to be a nonatomic join-semilattice, then the domain of entities will surely be infinite, whether countable or uncountable. It is a fact that a single sentence of natural language, albeit only finitely long, can talk about an infinite number of entities, as exemplified in (1).

a. Every natural number is odd or even.

b. All water is wet. (Link, 1983)

Given this, it is linguistically unjustified to assume the domain of entities to be finite. Since Partee (1986) herself discussed Link (1983), she was certainly aware that the domain of entities might very 
well be infinite, so it is unlikely that Partee followed van Benthem about the size of the domain of entities.

What difference does it make if the domain $D_{e}$ of entities is infinite, then? Fact 2 would be correct if "homomorphism" were read as "complete homomorphism." A complete Boolean homomorphism is a Boolean homomorphism that in addition preserves infinite joins and meets. It is clear from the equalities given in Partee's Fact 1 that she did not mean complete homomorphism by the word "homomorphism." When $D_{e}$ is finite, this does not matter since in that case, $D_{\langle\langle e, t\rangle, t\rangle}$ is also finite, and consequently, every Boolean homomorphism from $D_{\langle\langle e, t\rangle, t\rangle}$ is necessarily complete. However, when $D_{e}$ is infinite, so is $D_{\langle\langle e, t\rangle, t\rangle}$, and in that case, a Boolean homomorphism from $D_{\langle\langle e, t\rangle, t\rangle}$ can be incomplete, and "Fact" 2 turns out to be false.

This paper essentially consists of extended notes on Partee (1986). Section 2 shows that BE is the unique complete homomorphism that makes the Partee triangle commute and also that it is not the unique homomorphism that does so if $D_{e}$ is infinite. Section 3 discusses why it is important that $B E$ is complete by examining its interaction with $A$. Finally, Section 4 shows that $A$ is special among the many inverses of BE. The paper assumes the reader's basic familiarity with Boolean algebras and does not provide definitions or explanations of the technical terms that are used. I would suggest Givant and Halmos (2009) as a good general reference.

\section{Uniqueness and Nonuniqueness Proofs}

Since it is cumbersome to work with functions, let's adopt set talk. The operators lift, ident and $\mathrm{BE}$ and the Partee triangle can be rendered as follows, where $D=D_{e}$ is a nonempty set of entities and $\wp$ denotes power set. ${ }^{1}$

$$
\begin{aligned}
\text { lift } & =\lambda x .\{P \in \wp(D) \mid x \in P\}, \\
\text { ident } & =\lambda x .\{x\}, \\
\text { BE } & =\lambda \mathscr{P} .\{x \in D \mid\{x\} \in \mathscr{P}\} .
\end{aligned}
$$

\footnotetext{
${ }^{1}$ Here and below, $\lambda$ 's are used merely to describe functions; they are not meant to be symbols in a logical language that are to be interpreted.
}

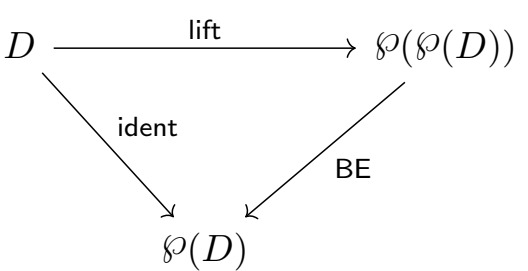

Diagram 2: The Partee triangle (set talk rendition)

Theorem 1. BE is a complete homomorphism from $\wp(\wp(D))$ to $\wp(D)$.

Proof. It suffices to show that BE preserves arbitrary unions and complements (denoted by ${ }^{\mathrm{C}}$ ). If $\left\{\mathscr{P}_{i}\right\}_{i \in I}$ is an arbitrary family in $\wp(\wp(D))$,

$$
\begin{aligned}
\operatorname{BE}\left(\bigcup_{i \in I} \mathscr{P}_{i}\right) & =\left\{x \in D \mid\{x\} \in \bigcup_{i \in I} \mathscr{P}_{i}\right\} \\
& =\bigcup_{i \in I}\left\{x \in D \mid\{x\} \in \mathscr{P}_{i}\right\} \\
& =\bigcup_{i \in I} \operatorname{BE}\left(\mathscr{P}_{i}\right) .
\end{aligned}
$$

For all $\mathscr{P} \in \wp(\wp(D))$,

$$
\begin{aligned}
\operatorname{BE}\left(\mathscr{P}^{\mathrm{c}}\right) & =\left\{x \in D \mid\{x\} \in \mathscr{P}^{\mathrm{c}}\right\} \\
& =\{x \in D \mid\{x\} \notin \mathscr{P}\} \\
& =\{x \in D \mid\{x\} \in \mathscr{P}\}^{\mathrm{c}} \\
& =\mathrm{BE}(\mathscr{P})^{\mathrm{c}} .
\end{aligned}
$$

Lemma 2. Let $h$ be a homomorphism from $\wp(\wp(D))$ to $\wp(D)$. The following conditions are equivalent.

(i) $h=\mathrm{BE}$.

(ii) For all $x \in D$ and all $\mathscr{P} \in \wp(\wp(D))$, if $x \in h(\mathscr{P})$ then $\{x\} \in \mathscr{P}$.

Proof. (i) $\Rightarrow$ (ii). Obvious since $x \in \mathrm{BE}(\mathscr{P})$ iff $\{x\} \in \mathscr{P}$.

(ii) $\Rightarrow$ (i). We show the contrapositive. Suppose $h \neq \mathrm{BE}$, so there exists some $\mathscr{P} \in \wp(\wp(D))$ such that

$$
h(\mathscr{P}) \neq \mathrm{BE}(\mathscr{P})=\{x \in D \mid\{x\} \in \mathscr{P}\} .
$$

Then, either there is some $a \in D$ such that $a \in$ $h(\mathscr{P})$ and $\{a\} \notin \mathscr{P}$ or there is some $a \in D$ such that $a \notin h(\mathscr{P})$ and $\{a\} \in \mathscr{P}$. In the latter case, we have $a \in h(\mathscr{P})^{\mathrm{c}}=h\left(\mathscr{P}^{\mathrm{c}}\right)$ and $\{a\} \notin \mathscr{P}^{\mathrm{c}}$. Thus, in either case, (ii) does not hold.

Lemma 3. Let $h$ be a homomorphism from $\wp(\wp(D))$ to $\wp(D)$ such that $h \circ$ lift $=$ ident. For any nonsingleton set $P \in \wp(D), h(\{P\})=\varnothing$. 
Proof. We first show that $h(\{\varnothing\})=\varnothing$. Assume that there exists some $a \in h(\{\varnothing\})$. Since $\varnothing \notin \operatorname{lift}(a)$, we have $\{\varnothing\} \subseteq \operatorname{lift}(a)^{c}$. Since $h$ is a homomorphism and hence preserves order,

$$
\begin{aligned}
h(\{\varnothing\}) & \subseteq h\left(\operatorname{lift}(a)^{\mathrm{c}}\right) \\
& =h(\operatorname{lift}(a))^{\mathrm{c}} \\
& =(h \circ \operatorname{lift})(a)^{\mathrm{c}} \\
& =\operatorname{ident}(a)^{\mathrm{c}} \\
& =\{a\}^{\mathrm{c}},
\end{aligned}
$$

so $a \notin h(\{\varnothing\})$, a contradiction.

Next, we show that $h(\{P\})=\varnothing$ if $|P| \geq 2$. Assume that for some $P$ with $|P| \geq 2$, there exists some $a \in h(\{P\})$. Since $|P| \geq 2$, there is some $b \in P$ with $b \neq a$. Since $P \in \operatorname{lift}(b)$ and hence $\{P\} \subseteq \operatorname{lift}(b)$, we have

$$
\begin{aligned}
h(\{P\}) & \subseteq h(\operatorname{lift}(b)) \\
& =(h \circ \operatorname{lift})(b) \\
& =\operatorname{ident}(b) \\
& =\{b\} .
\end{aligned}
$$

Since $a \in h(\{P\})$, we obtain $b=a$, a contradiction.

Theorem 4. BE is the unique complete homomorphism that makes the Partee triangle commute.

Proof. To see that BE makes the Partee triangle commute, observe that for any $a \in D$,

$$
\begin{aligned}
& \mathrm{BE}(\operatorname{lift}(a)) \\
& =\{x \in D \mid\{x\} \in \operatorname{lift}(a)\} \\
& =\{x \in D \mid\{x\} \in\{P \in \wp(D) \mid a \in P\}\} \\
& =\{x \in D \mid a \in\{x\}\} \\
& =\{x \in D \mid x=a\} \\
& =\{a\} \\
& =\operatorname{ident}(a) .
\end{aligned}
$$

Now, let $h$ be a complete homomorphism from $\wp(\wp(D))$ to $\wp(D)$ such that $h \circ$ lift $=$ ident. We show that $h=$ BE. Let $a \in D$ and $\mathscr{P} \in \wp(\wp(D))$ satisfy $a \in h(\mathscr{P})$. By Lemma 2 , it is sufficient to show that $\{a\} \in \mathscr{P}$. Since $h$ is a complete homomorphism,

$$
\begin{aligned}
\bigcup_{P \in \mathscr{P} \cap \operatorname{lift}(a)} h(\{P\}) & =h\left(\bigcup_{P \in \mathscr{P} \cap \operatorname{lift}(a)}\{P\}\right) \\
& =h(\mathscr{P} \cap \operatorname{lift}(a)) \\
& =h(\mathscr{P}) \cap h(\operatorname{lift}(a)) \\
& =h(\mathscr{P}) \cap \operatorname{ident}(a) \\
& =h(\mathscr{P}) \cap\{a\} \\
& =\{a\} .
\end{aligned}
$$

It follows that for some $P \in \mathscr{P} \cap \operatorname{lift}(a)$,

$$
h(\{P\})=\{a\} .
$$

By Lemma 3, $P$ must be a singleton set. Since the only singleton set contained in $\operatorname{lift}(a)$ is $\{a\}$, we have $\{a\}=P \in \mathscr{P} \cap \operatorname{lift}(a)$, so $\{a\} \in \mathscr{P}$.

Note that Theorem 4 immediately follows from Keenan and Faltz's (1985) Justification Theorem (p. 92) as well. Individuals in Keenan and Faltz's theory can be identified with the elements of the set $\left\{I_{x} \mid x \in D\right\}$, where $I_{x}=\operatorname{lift}(x)$. Given a function $f$ from the set of individuals into $\wp(D)$ such that $f\left(I_{x}\right)=\operatorname{ident}(x)$ for all $x \in D$, the Justification Theorem says that there exists a unique complete homomorphism from $\wp(\wp(D))$ to $\wp(D)$ that extends $f$.

When $D$ is finite, a homomorphism from $\wp(\wp(D))$ to $\wp(D)$ is necessarily a complete homomorphism, so by Theorem 4, BE is automatically the unique homomorphism that makes the Partee triangle commute. This is not the case, however, when $D$ is infinite. To consider such cases, the following lemma plays an important role of giving (unique) representations of homomorphisms that make the Partee triangle commute.

Lemma 5. Let h be a function from $\wp(\wp(D))$ into $\wp(D)$. The following conditions are equivalent.

(i) $h$ is a homomorphism from $\wp(\wp(D))$ to $\wp(D)$ and $h \circ$ lift $=$ ident.

(ii) There is a family $\left\{\mathfrak{U}_{x}\right\}_{x \in D}$ of subsets of $\wp(\wp(D))$ such that each $\mathfrak{U}_{x}$ is an ultrafilter in the Boolean algebra $\wp(\operatorname{lift}(x))$ satisfying $\operatorname{lift}(x) \cap \operatorname{lift}(y) \notin \mathfrak{U}_{x}$ for all $y \neq x$, and

$$
h=\lambda \mathscr{P} .\left\{x \in D \mid \mathscr{P} \cap \operatorname{lift}(x) \in \mathfrak{U}_{x}\right\} .
$$

Proof. (i) $\Rightarrow$ (ii). Assume (i). For each $x \in D$, let

$$
\mathfrak{U}_{x}=\{\mathscr{P} \in \wp(\operatorname{lift}(x)) \mid x \in h(\mathscr{P})\} .
$$


To begin with, we show that $\mathfrak{U}_{x}$ is an ultrafilter in the Boolean algebra $\wp(\operatorname{lift}(x))$. First, since $x \in\{x\}=\operatorname{ident}(x)=h(\operatorname{lift}(x))$, the top element lift $(x)$ of $\wp(\operatorname{lift}(x))$ belongs to $\mathfrak{U}_{x}$. Second, if $\mathscr{P}, \mathscr{Q} \in \mathfrak{U}_{x}$, then $x \in h(\mathscr{P})$ and $x \in h(\mathscr{Q})$ and hence $x \in h(\mathscr{P}) \cap h(\mathscr{Q})=h(\mathscr{P} \cap \mathscr{Q})$, so $\mathscr{P} \cap \mathscr{Q} \in \mathfrak{U}_{x}$. Third, if $\mathscr{P} \in \mathfrak{U}_{x}$ and $\mathscr{Q} \in$ $\wp(\operatorname{lift}(x))$ satisfy $\mathscr{P} \subseteq \mathscr{Q}$, then $x \in h(\mathscr{P}) \subseteq$ $h(\mathscr{Q})$, so $\mathscr{Q} \in \mathfrak{U}_{x}$. This establishes that $\mathfrak{U}_{x}$ is a filter in $\wp(\operatorname{lift}(x))$. To see that $\mathfrak{U}_{x}$ is an ultrafilter, suppose $\mathscr{P} \in \wp(\operatorname{lift}(x))$ and $\mathscr{P} \notin \mathfrak{U}_{x}$. Since $x \in h(\operatorname{lift}(x))=h(\mathscr{P} \cup(\mathscr{P} \mathrm{c} \cap \operatorname{lift}(x)))=$ $h(\mathscr{P}) \cup h\left(\mathscr{P}^{\mathrm{c}} \cap \operatorname{lift}(x)\right)$ and $x \notin h(\mathscr{P})$, we have $x \in h\left(\mathscr{P}^{\mathrm{c}} \cap \operatorname{lift}(x)\right)$, so $\mathscr{P}^{\mathrm{c}} \cap \operatorname{lift}(x) \in \mathfrak{U}_{x}$. Thus, the complement of $\mathscr{P}$ in $\wp(\operatorname{lift}(x))$ belongs to $\mathfrak{U}_{x}$.

Next, observe that if lift $(x) \cap \operatorname{lift}(y) \in \mathfrak{U}_{x}$, then $x \in h(\operatorname{lift}(x) \cap \operatorname{lift}(y))=h(\operatorname{lift}(x)) \cap h(\operatorname{lift}(y))$, so $x \in h(\operatorname{lift}(y))=\operatorname{ident}(y)=\{y\}$ and therefore $x=y$. It follows that $\operatorname{lift}(x) \cap \operatorname{lift}(y) \notin \mathfrak{U}_{x}$ for all $y \neq x$. This establishes that the family $\left\{\mathfrak{U}_{x}\right\}_{x \in D}$ has the desired properties.

Now, for all $\mathscr{P} \in \wp(\wp(D))$, we have

$$
\begin{aligned}
h\left(\mathscr{P} \cap \operatorname{lift}(x)^{\mathrm{c}}\right) & \subseteq h\left(\operatorname{lift}(x)^{\mathrm{c}}\right) \\
& =h(\operatorname{lift}(x))^{\mathrm{c}} \\
& =\operatorname{ident}(x)^{\mathrm{c}} \\
& =\{x\}^{\mathrm{c}},
\end{aligned}
$$

so $x \notin h\left(\mathscr{P} \cap \operatorname{lift}(x)^{\mathrm{c}}\right)$. It follows that

$$
\begin{array}{ll} 
& x \in h(\mathscr{P}) \\
\text { iff } & x \in h\left((\mathscr{P} \cap \operatorname{lift}(x)) \cup\left(\mathscr{P} \cap \operatorname{lift}(x)^{\mathrm{c}}\right)\right) \\
\text { iff } & x \in h(\mathscr{P} \cap \operatorname{lift}(x)) \cup h\left(\mathscr{P} \cap \operatorname{lift}(x)^{\mathrm{c}}\right) \\
\text { iff } & x \in h(\mathscr{P} \cap \operatorname{lift}(x)) \\
\text { iff } & \mathscr{P} \cap \operatorname{lift}(x) \in \mathfrak{U}_{x} .
\end{array}
$$

Thus $h(\mathscr{P})=\left\{x \in D \mid \mathscr{P} \cap \operatorname{lift}(x) \in \mathfrak{U}_{x}\right\}$.

(ii) $\Rightarrow$ (i). Assume (ii). To show that $h$ is a homomorphism, it suffices to check that it preserves finite union and complement. Being an ultrafilter, $\mathfrak{U}_{x}$ is a prime filter. Therefore, for all $\mathscr{P}, \mathscr{Q} \in \wp(\wp(D))$,

$$
\begin{array}{ll} 
& x \in h(\mathscr{P} \cup \mathscr{Q}) \\
\text { iff } & (\mathscr{P} \cup \mathscr{Q}) \cap \operatorname{lift}(x) \in \mathfrak{U}_{x} \\
\text { iff } & (\mathscr{P} \cap \operatorname{lift}(x)) \cup(\mathscr{Q} \cap \operatorname{lift}(x)) \in \mathfrak{U}_{x} \\
\text { iff } & \mathscr{P} \cap \operatorname{lift}(x) \in \mathfrak{U}_{x} \text { or } \mathscr{Q} \cap \operatorname{lift}(x) \in \mathfrak{U}_{x} \\
\text { iff } & x \in h(\mathscr{P}) \text { or } x \in h(\mathscr{Q}) \\
\text { iff } & x \in h(\mathscr{P}) \cup h(\mathscr{Q}),
\end{array}
$$

so $h(\mathscr{P} \cup \mathscr{Q})=h(\mathscr{P}) \cup h(\mathscr{Q})$. Also, for all $\mathscr{P} \in \wp(\wp(D))$, since $\mathscr{P} \cap \operatorname{lift}(x)$ and $\mathscr{P}^{c} \cap \operatorname{lift}(x)$ are complements of each other in $\wp(\operatorname{lift}(x))$ and since $\mathfrak{U}_{x}$ is an ultrafilter in $\wp(\operatorname{lift}(x))$,

$$
\begin{aligned}
x \in h\left(\mathscr{P}^{\mathrm{c}}\right) \quad \text { iff } & \mathscr{P}^{\mathrm{c}} \cap \operatorname{lift}(x) \in \mathfrak{U}_{x} \\
\text { iff } & \mathscr{P} \cap \operatorname{lift}(x) \notin \mathfrak{U}_{x} \\
\text { iff } & x \notin h(\mathscr{P}) \\
\text { iff } & x \in h(\mathscr{P})^{\mathrm{c}},
\end{aligned}
$$

so $h\left(\mathscr{P}^{\mathrm{c}}\right)=h(\mathscr{P})^{\mathrm{c}}$.

It remains to show that $h \circ$ lift $=$ ident. While $\operatorname{lift}(x) \cap \operatorname{lift}(y) \notin \mathfrak{U}_{x}$ for every $y \neq x$, we have $\operatorname{lift}(x) \cap \operatorname{lift}(x)=\operatorname{lift}(x) \in \mathfrak{U}_{x}$ since an ultrafilter in $\wp(\operatorname{lift}(x))$ contains the top element of $\wp(\operatorname{lift}(x))$. Consequently,

$$
\begin{aligned}
h(\operatorname{lift}(x)) & =\left\{y \in D \mid \operatorname{lift}(x) \cap \operatorname{lift}(y) \in \mathfrak{U}_{y}\right\} \\
& =\{x\} \\
& =\operatorname{ident}(x) .
\end{aligned}
$$

Lemma 6. Let $\mathfrak{U}_{x}$ be a principal ultrafilter in $\wp(\operatorname{lift}(x))$. The following conditions are equivalent.

(i) $\operatorname{lift}(x) \cap \operatorname{lift}(y) \notin \mathfrak{U}_{x}$ for all $y \neq x$.

(ii) $\mathfrak{U}_{x}$ is generated by $\{\{x\}\}$.

Proof. (i) $\Rightarrow$ (ii). Assume (i). Since $\mathfrak{U}_{x}$ is a principal filter in $\wp(\operatorname{lift}(x))$, there is some $\mathscr{Q} \in$ $\wp(\operatorname{lift}(x))$ that generates it, i.e.,

$$
\mathfrak{U}_{x}=\uparrow \mathscr{Q}=\{\mathscr{P} \in \wp(\operatorname{lift}(x)) \mid \mathscr{Q} \subseteq \mathscr{P}\} .
$$

We show that $\mathscr{Q}=\{\{x\}\}$. For every $y \neq x$, we have $\operatorname{lift}(x) \cap \operatorname{lift}(y) \notin \mathfrak{U}_{x}$, and because $\mathfrak{U}_{x}$ is an ultrafilter, this implies that its complement $\operatorname{lift}(x) \cap \operatorname{lift}(y)^{\mathrm{c}}$ in $\wp(\operatorname{lift}(x))$ belongs to $\mathfrak{U}_{x}$, so $\mathscr{Q} \subseteq \operatorname{lift}(x) \cap \operatorname{lift}(y)^{\mathrm{c}}$. It follows that

$$
\begin{aligned}
\mathscr{Q} \subseteq & \bigcap_{y \neq x}\left(\operatorname{lift}(x) \cap \operatorname{lift}(y)^{\mathrm{c}}\right) \\
= & \operatorname{lift}(x) \cap\left(\bigcap_{y \neq x} \operatorname{lift}(y)^{\mathrm{c}}\right) \\
= & \{P \in \wp(D) \mid x \in P\} \\
& \cap\left(\bigcap_{y \neq x}\{P \in \wp(D) \mid y \in P\}^{\mathrm{c}}\right) \\
= & \{P \in \wp(D) \mid x \in P\} \\
& \cap\left(\bigcap_{y \neq x}\{P \in \wp(D) \mid y \notin P\}\right) \\
= & \{P \in \wp(D) \mid x \in P \text { and } y \notin P \text { for all } y \neq x\} \\
= & \{\{x\}\} .
\end{aligned}
$$

Since $\mathfrak{U}_{x}=\uparrow \mathscr{Q}$ is an ultrafilter, $\mathscr{Q} \neq \varnothing$. Hence $\mathscr{Q}=\{\{x\}\}$. 
(ii) $\Rightarrow$ (i). Assume $\mathfrak{U}_{x}=\uparrow\{\{x\}\}$, i.e.,

$$
\begin{aligned}
\mathfrak{U}_{x} & =\{\mathscr{P} \in \wp(\operatorname{lift}(x)) \mid\{\{x\}\} \subseteq \mathscr{P}\} \\
& =\{\mathscr{P} \in \wp(\operatorname{lift}(x)) \mid\{x\} \in \mathscr{P}\} .
\end{aligned}
$$

Since

$$
\begin{aligned}
& \operatorname{lift}(x) \cap \operatorname{lift}(y) \\
& =\{P \in \wp(D) \mid x \in P\} \cap\{P \in \wp(D) \mid y \in P\} \\
& =\{P \in \wp(D) \mid\{x, y\} \subseteq P\},
\end{aligned}
$$

if $y \neq x$, then $\{x\} \notin \operatorname{lift}(x) \cap \operatorname{lift}(y)$, so $\operatorname{lift}(x) \cap$ $\operatorname{lift}(y) \notin \mathfrak{U}_{x}$.

Lemma 7. Let $D$ be infinite. For every $x \in$ $D$, there exists a nonprincipal ultrafilter $\mathfrak{U}_{x}$ in $\wp(\operatorname{lift}(x))$ such that $\operatorname{lift}(x) \cap \operatorname{lift}(y) \notin \mathfrak{U}_{x}$ for all $y \neq x$.

Proof. For $x \in D$, let $\mathfrak{E}_{x}$ be the following subset of $\wp(\operatorname{lift}(x))$ :

$$
\begin{aligned}
\mathfrak{E}_{x}= & \{\{\{x\}\}\} \cup \\
& \{\operatorname{lift}(x) \cap \operatorname{lift}(y) \mid y \in D \text { and } y \neq x\} .
\end{aligned}
$$

If $\mathfrak{F}$ is a finite subset of $\mathfrak{E}_{x}$, then since $D$ is infinite, there is some $y \in D$ such that $\operatorname{lift}(x) \cap \operatorname{lift}(y) \notin \mathfrak{F}$, so in particular $\{x, y\} \notin \bigcup \mathfrak{F}$ and thus $\bigcup \mathfrak{F}$ cannot equal $\operatorname{lift}(x)$, the top element of $\wp(\operatorname{lift}(x))$. $\mathfrak{E}_{x}$ thus has the finite join property, so the ideal $\mathfrak{I}_{x}$ generated by $\mathfrak{E}_{x}$ in $\wp(\operatorname{lift}(x))$ is proper. By the Boolean prime ideal theorem, $\mathfrak{I}_{x}$ can be extended to a prime ideal, i.e., a maximal ideal $\mathfrak{M}_{x}$ in $\wp(\operatorname{lift}(x)) .^{2}$ Let $\mathfrak{U}_{x}$ be the dual ultrafilter of $\mathfrak{M}_{x}$ in $\wp(\operatorname{lift}(x))$ :

$$
\mathfrak{U}_{x}=\left\{\mathscr{P}^{\mathrm{c}} \cap \operatorname{lift}(x) \mid \mathscr{P} \in \mathfrak{M}_{x}\right\} .
$$

For all $y \neq x$, since $\operatorname{lift}(x) \cap \operatorname{lift}(y) \in \mathfrak{E}_{x} \subseteq \mathfrak{M}_{x}$, we have $\operatorname{lift}(x) \cap \operatorname{lift}(y) \notin \mathfrak{U}_{x}$. Since $\{\{x\}\} \in$ $\mathfrak{E}_{x} \subseteq \mathfrak{M}_{x}$, we also have $\{\{x\}\} \notin \mathfrak{U}_{x}$. Lemma 6 then implies that $\mathfrak{U}_{x}$ is not a principal filter.

Theorem 8. If $D$ is infinite, there are uncountably many homomorphisms $h$ from $\wp(\wp(D))$ to $\wp(D)$ such that $h \circ$ lift $=$ ident.

Proof. Let $D$ be infinite. By Lemma 5, a homomorphism $h$ from $\wp(\wp(D))$ to $\wp(D)$ such that $h \circ$ lift $=$ ident is written

$$
h=\lambda \mathscr{P} .\left\{x \in D \mid \mathscr{P} \cap \operatorname{lift}(x) \in \mathfrak{U}_{x}\right\},
$$

\footnotetext{
${ }^{2}$ Thus Lemma 7 (and hence also Theorem 8) uses the Boolean prime ideal theorem, a weaker form of the axiom of choice.
}

where $\mathfrak{U}_{x}$ is an ultrafilter in $\wp(\operatorname{lift}(x))$ such that $\operatorname{lift}(x) \cap \operatorname{lift}(y) \notin \mathfrak{U}_{x}$ for all $y \neq x$. By Lemmata 6 and 7, there are at least two such ultrafilters $\mathfrak{U}_{x}$ for each $x \in D$ : a principal one and a nonprincipal one. For each $x$, different choices for $\mathfrak{U}_{x}$ clearly give rise to different homomorphisms. It follows that the cardinality of the set of homomorphisms $h$ such that $h \circ$ lift $=$ ident is at least $2^{|D|}$.

Observe that since

$$
\begin{array}{rll}
\{x\} \in \mathscr{P} \quad \text { iff } & \{\{x\}\} \subseteq \mathscr{P} \\
\text { iff } & \{\{x\}\} \subseteq \mathscr{P} \cap \operatorname{lift}(x) \\
\text { iff } & \mathscr{P} \cap \operatorname{lift}(x) \in \uparrow\{\{x\}\},
\end{array}
$$

we can write

$$
\begin{aligned}
\mathrm{BE} & =\lambda \mathscr{P} .\{x \in D \mid\{x\} \in \mathscr{P}\} \\
& =\lambda \mathscr{P} .\{x \in D \mid \mathscr{P} \cap \operatorname{lift}(x) \in \uparrow\{\{x\}\}\} .
\end{aligned}
$$

Thus, in Lemma 5's representation of BE, each $\mathfrak{U}_{x}$ is a principal filter in $\wp(\operatorname{lift}(x))$. This also explains why $\mathrm{BE}$ has to be the unique homomorphism that makes the Partee triangle commute when $D$ is finite, because in that case, each $\wp(\operatorname{lift}(x))$ is finite, and every filter in a finite Boolean algebra is necessarily principal.

Now suppose $D$ is infinite. By Theorem 8 , there is a homomorphism $h \neq \mathrm{BE}$ that makes the Partee triangle commute. By Theorem 4, we know that $h$ is not a complete homomorphism. It may be illuminating to confirm this fact directly. The observation in the previous paragraph implies that in Lemma 5's representation of $h$, there is some $a \in D$ such that $\mathfrak{U}_{a}$ is a nonprincipal ultrafilter in $\wp(\operatorname{lift}(a))$. We have $\{\{a\}\} \cap \operatorname{lift}(a)=\{\{a\}\} \notin \mathfrak{U}_{a}$ because $\{\{a\}\} \in \mathfrak{U}_{a}$ would imply $\mathfrak{U}_{a}=\uparrow\{\{a\}\}$ but $\mathfrak{U}_{a}$ is nonprincipal. Also, for all $x \neq a$, we have $\{\{a\}\} \cap \operatorname{lift}(x)=\varnothing \notin \mathfrak{U}_{x}$ because an ultrafilter does not contain the bottom element. Thus

$h(\{\{a\}\})=\left\{x \in D \mid\{\{a\}\} \cap \operatorname{lift}(x) \in \mathfrak{U}_{x}\right\}=\varnothing$.

Since $\{\{a\}\}$ is the only singleton set in $\operatorname{lift}(a)$, for every $P \in \operatorname{lift}(a)$ such that $P \neq\{\{a\}\}$, we have $h(\{P\})=\varnothing$ by Lemma 3. It follows that

$$
\bigcup_{P \in \operatorname{lift}(a)} h(\{P\})=\bigcup_{P \in \operatorname{lift}(a)} \varnothing=\varnothing .
$$

On the other hand,

$h\left(\bigcup_{P \in \operatorname{lift}(a)}\{P\}\right)=h(\operatorname{lift}(a))=\operatorname{ident}(a)=\{a\}$.

Thus

$$
h\left(\bigcup_{P \in \operatorname{lift}(a)}\{P\}\right) \neq \bigcup_{P \in \operatorname{lift}(a)} h(\{P\}) .
$$

So $h$ does not generally preserve an infinite union. 


\section{Why do we need a complete homomorphism?}

Partee (1986) proposes that BE is a type-shifting operator naturally employed in natural language semantics on the grounds that it is a Boolean homomorphism and it makes the Partee triangle commute. As we have seen, however, when $D$ is infinite, there are infinitely many such homomorphisms. Couldn't they then perhaps be employed as type-shifting operators in place of BE? What distinguishes $B E$ from all the rest is the fact that it is the only complete one. So the question boils down to this: how should being a complete homomorphism matter?

To answer this question, let's recall Partee's (1986) discussion of the functions THE and A from $D_{\langle e, t\rangle}$ into $D_{\langle\langle e, t\rangle, t\rangle}$, which in set talk can be rendered as the following functions from $\wp(D)$ into $\wp(\wp(D))$.

$$
\begin{aligned}
\mathrm{THE} & =\lambda P .\{Q \in \wp(D)|| P \mid=1 \text { and } P \subseteq Q\}, \\
\mathrm{A} & =\lambda P .\{Q \in \wp(D) \mid P \cap Q \neq \varnothing\} .
\end{aligned}
$$

Partee argues that THE and A are "natural" since they are inverses of BE in the sense that for all $P \in \wp(D)$,

$$
\begin{aligned}
\mathrm{BE}(\operatorname{THE}(P)) & = \begin{cases}P & \text { if } P \text { is a singleton, } \\
\varnothing & \text { otherwise },\end{cases} \\
\mathrm{BE}(\mathrm{A}(P)) & =P
\end{aligned}
$$

One should then wonder whether analogous equalities hold with other homomorphisms that make the Partee triangle commute.

It is immediate that an analogous equality holds with THE.

Theorem 9. Let $h$ be a homomorphism from $\wp(\wp(D))$ to $\wp(D)$ such that $h \circ$ lift $=$ ident. For all $P \in \wp(D)$,

$$
h(\operatorname{THE}(P))= \begin{cases}P & \text { if } P \text { is a singleton, } \\ \varnothing \quad \text { otherwise. }\end{cases}
$$

Proof. For any $x \in D, \operatorname{THE}(\{x\})=\operatorname{lift}(x)$, so $h(\operatorname{THE}(\{x\}))=h(\operatorname{lift}(x))=\operatorname{ident}(x)=\{x\}$. If $P \in \wp(D)$ is not a singleton, then $\operatorname{THE}(P)=\varnothing$, so $h(\operatorname{THE}(P))=h(\varnothing)=\varnothing$.

With $\mathrm{A}$, by contrast, an analogous equality does not generally hold, and this is where (in)completeness becomes crucial.
Theorem 10. Let $h$ be a homomorphism from $\wp(\wp(D))$ to $\wp(D)$ such that $h \circ$ lift $=$ ident. For all $P \in \wp(D)$,

$$
h(\mathrm{~A}(P)) \supseteq P .
$$

In particular, if $P$ is finite,

$$
h(\mathrm{~A}(P))=P .
$$

Proof. Let $P \in \wp(D)$. We have

$$
\begin{aligned}
\mathrm{A}(P) & =\{Q \in \wp(D) \mid P \cap Q \neq \varnothing\} \\
& =\bigcup_{x \in P}\{Q \in \wp(D) \mid x \in Q\} \\
& =\bigcup_{x \in P} \operatorname{lift}(x) .
\end{aligned}
$$

Thus, for every $x \in P$, lift $(x) \subseteq \mathrm{A}(P)$ and hence $\{x\}=\operatorname{ident}(x)=h(\operatorname{lift}(x)) \subseteq h(\mathrm{~A}(P))$. Thus

$$
P=\bigcup_{x \in P}\{x\} \subseteq h(\mathrm{~A}(P)) .
$$

If $P$ is finite, then the homomorphism properties of $h$ ensure that

$$
\begin{aligned}
h(\mathrm{~A}(P)) & =h\left(\bigcup_{x \in P} \operatorname{lift}(x)\right) \\
& =\bigcup_{x \in P} h(\operatorname{lift}(x)) \\
& =\bigcup_{x \in P} \operatorname{ident}(x) \\
& =\bigcup_{x \in P}\{x\} \\
& =P .
\end{aligned}
$$

Theorem 10 suggests that homomorphisms other than $B E$ are undesirable as a type-shifting operator to replace BE, even if they make the Partee triangle commute. To see this point, imagine that some such homomorphism $h \neq \mathrm{BE}$ were actually employed as a type-shifter.

First, consider the following example.

$$
\text { André is a girl. }
$$

Following Partee (1986), let's assume that the verb $b e$ is semantically vacuous and a type-shifter is inserted to convert a quantifier into a predicate. (2) would then be analyzed as

$$
\text { André } \in h(\llbracket \text { a girl } \rrbracket)=h(\mathrm{~A}(\llbracket \operatorname{girl} \rrbracket)) .
$$

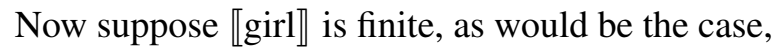
say $\llbracket$ girl $\rrbracket=\{$ Mari, Meiko, Hana $\}$. Then by Theorem 10 ,

$$
h(\mathrm{~A}(\llbracket \operatorname{girl} \rrbracket))=\llbracket \operatorname{girl} \rrbracket=\{\text { Mari, Meiko, Hana }\},
$$

so (3) is equivalent to 


$$
\text { André } \in\{\text { Mari, Meiko, Hana }\},
$$

or what amounts to the same thing,

$$
\begin{aligned}
& \text { André }=\text { Mari or } \\
& \text { André }=\text { Meiko or } \\
& \text { André }=\text { Hana } .
\end{aligned}
$$

These are indeed the desired truth conditions for (2), so no problem arises in this case.

Now consider the examples in (6).

$$
\begin{aligned}
& \text { a. } \pi \text { is a natural number. } \\
& \text { b. This is some water. }
\end{aligned}
$$

These would be analyzed as in (7), assuming that this denotes an entity and that $\llbracket$ some $\rrbracket=\llbracket \mathrm{a} \rrbracket=\mathrm{A}$.

$$
\begin{array}{ll}
\text { a. } & \pi \in h(\mathrm{~A}(\llbracket \text { natural number } \rrbracket)) \\
\text { b. } & \llbracket \text { this } \rrbracket \in h(\mathrm{~A}(\llbracket \text { water } \rrbracket)) .
\end{array}
$$

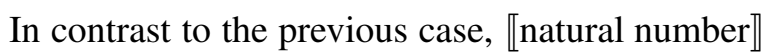

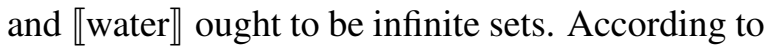
Theorem 10, what we can know is then only that

$$
\begin{aligned}
h(\mathrm{~A}(\llbracket \text { natural number } \rrbracket)) & \supseteq \llbracket \text { natural number } \rrbracket, \\
h(\mathrm{~A}(\llbracket \text { water } \rrbracket)) & \supseteq \llbracket \text { water } \rrbracket .
\end{aligned}
$$

What these inequalities imply is that even though $\pi \notin \llbracket$ natural number $\rrbracket,(7-a)$ might hold and hence

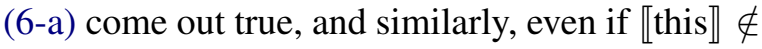

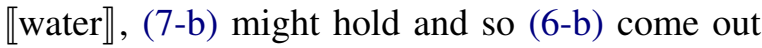
true. Such states of affairs would be clearly undesirable. This suggests that $h$ should not be used as a type-shifter in natural language semantics.

The above argument does not show, however, that undesirable states of affairs necessarily ensue, as the inequality $h(\mathrm{~A}(P)) \supseteq P$ in Theorem 10 is not necessarily a proper inclusion. Then, even in a case where $D$ is infinite, might there perhaps be a homomorphism $h \neq \mathrm{BE}$ such that $h(\mathrm{~A}(P))=P$ for all $P \in \wp(D)$ ? The following theorem tells us that this possibility never obtains. Note that it also characterizes BE without directly mentioning completeness or the property of making the Partee triangle commute.

Theorem 11. BE is the unique homomorphism $h$ from $\wp(\wp(D))$ to $\wp(D)$ such that $h \circ \mathrm{A}$ is the identity map on $\wp(D)$.

Proof. Since BE is a complete homomorphism, by substituting BE for $h$ in the last set of equalities in the proof of Theorem 10, we can see that $\mathrm{BE}(\mathrm{A}(P))=P$ for all $P \in \wp(D)$.
To show the uniqueness, assume to the contrary that there is a homomorphism $h \neq \mathrm{BE}$ such that $h \circ \mathrm{A}$ is the identity map. By Lemma 2 , for some $a \in D$ and some $\mathscr{P} \in \wp(\wp(D))$, we have $a \in$ $h(\mathscr{P})$ and $\{a\} \notin \mathscr{P}$. Since

$$
\begin{aligned}
\mathrm{A}(D \backslash\{a\}) & =\{Q \in \wp(D) \mid(D \backslash\{a\}) \cap Q \neq \varnothing\} \\
& =\{Q \in \wp(D) \mid Q \backslash\{a\} \neq \varnothing\} \\
& =\{Q \in \wp(D) \mid Q \neq\{a\}, \varnothing\} \\
& =\wp(D) \backslash\{\{a\}, \varnothing\}
\end{aligned}
$$

and since $\{a\} \notin \mathscr{P}$, we have

$$
\mathscr{P} \subseteq \wp(D) \backslash\{\{a\}\}=\mathrm{A}(D \backslash\{a\}) \cup\{\varnothing\} .
$$

Since $h \circ \mathrm{A}$ is the identity map, it follows that

$$
\begin{aligned}
h(\mathscr{P}) & \subseteq h(\mathrm{~A}(D \backslash\{a\}) \cup\{\varnothing\}) \\
& =h(\mathrm{~A}(D \backslash\{a\})) \cup h(\{\varnothing\}) \\
& =(D \backslash\{a\}) \cup h(\{\varnothing\}) \\
& =(D \backslash\{a\}) \cup \varnothing \quad \text { (by } \\
& =D \backslash\{a\} .
\end{aligned}
$$

This contradicts $a \in h(\mathscr{P})$.

It follows from Theorems 10 and 11 that if $h \neq \mathrm{BE}$ is a homomorphism from $\wp(\wp(D))$ to $\wp(D)$ and $h \circ$ lift $=$ ident, then there exists some infinite set $P \in \wp(D)$ such that $h(\mathrm{~A}(P)) \supsetneq P$. Indeed, we can find a concrete example. Since $h \neq \mathrm{BE}$, in Lemma 5's representation, there is some $a \in D$ such that $\mathfrak{U}_{a}$ is a nonprincipal ultrafilter in $\wp(\operatorname{lift}(a))$. We have $\{\{a\}\} \notin \mathfrak{U}_{a}$ since $\mathfrak{U}_{a}$ is nonprincipal. Since

$$
\begin{aligned}
\mathrm{A}(D \backslash\{a\}) \cap \operatorname{lift}(a) & =(\wp(D) \backslash\{\{a\}, \varnothing\}) \cap \operatorname{lift}(a) \\
& =\{\{a\}\}^{\mathrm{c}} \cap \operatorname{lift}(a)
\end{aligned}
$$

is the complement of $\{\{a\}\}$ in $\wp(\operatorname{lift}(a))$ and since $\mathfrak{U}_{a}$ is an ultrafilter in $\wp(\operatorname{lift}(a))$, we have

$$
\mathrm{A}(D \backslash\{a\}) \cap \operatorname{lift}(a) \in \mathfrak{U}_{a} .
$$

According to Lemma 5, this implies that

$$
a \in h(\mathrm{~A}(D \backslash\{a\})) .
$$

Combining with Theorem 10, we conclude that

$$
h(\mathrm{~A}(D \backslash\{a\}))=D \supsetneq D \backslash\{a\} .
$$

The discussion in this section is just another example demonstrating the significance of the notion of completeness of the Boolean structures and homomorphisms between them that are employed in natural language semantics, which was extensively argued for by Keenan and Faltz (1985). 


\section{Inverses of $B E$}

Having discussed the naturalness of BE, Partee (1986) asks what possible determiners $\delta$ are inverses of $\mathrm{BE}$, i.e., $\mathrm{BE}(\delta(P))=P$ for all $P \in$ $\wp(D)$. It is immediate that a necessary and sufficient condition for $\delta$ to be an inverse of $\mathrm{BE}$ is that

$$
\begin{aligned}
& \text { for all } P \in \wp(D), \\
& \{x \in D \mid\{x\} \in \delta(P)\}=P,
\end{aligned}
$$

so there exist many inverses of $B E$. A is one but

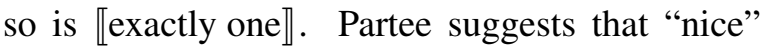
formal properties such as being increasing (in both arguments) and being symmetric might distinguish $A$ from the others. Contrary to her claim, though, symmetry fails to distinguish $A$ from

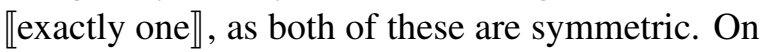
the other hand, the property of being increasing

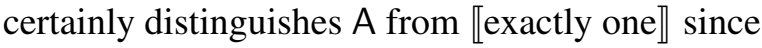

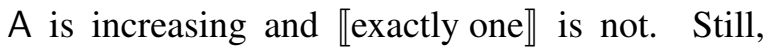
there are many inverses of BE other than $A$ that are increasing, as the reader can easily check. Then, how might formal properties single A out?

At this point, we shall recall Keenan and Stavi's (1986) view that all possible determiners are expressible as Boolean combinations of "basic" determiners, which are all increasing and weakly conservative. $^{3}$ These two properties are defined as follows, where $\delta$ is an arbitrary function from $\wp(D)$ into $\wp(\wp(D))$.

$$
\begin{aligned}
& \delta \text { is increasing } \\
& \Leftrightarrow \text { for all } P, Q_{1}, Q_{2} \in \wp(D) \text {, if } Q_{1} \in \delta(P) \\
& \text { and } Q_{1} \subseteq Q_{2} \text {, then } Q_{2} \in \delta(P) . \\
& \delta \text { is weakly conservative } \\
& \Leftrightarrow \text { for all } P, Q \in \wp(D) \text {, if } Q \in \delta(P) \text { then } \\
& P \cap Q \in \delta(P) .
\end{aligned}
$$

Keenan and Stavi (1986) proved that the functions obtained as Boolean combinations of basic determiners are exactly those functions from $\wp(D)$ into $\wp(\wp(D))$ that are conservative:

$$
\begin{aligned}
& \delta \text { is conservative } \\
& \Leftrightarrow \text { for all } P, Q \in \wp(D), Q \in \delta(P) \text { iff } \\
& P \cap Q \in \delta(P) .
\end{aligned}
$$

Keenan and Stavi proposed that this accounts for

\footnotetext{
${ }^{3}$ In Keenan and Stavi's (1986) theory, the determiner no, which is not increasing, is not a basic determiner. Keenan and Stavi suggest that increasingness may be a universal property of monomorphemic determiners, if negative determiners like no are analyzed as bimorphemic, consisting of a negative morpheme $N$ - and a stem.
}

the apparent fact that all determiners are conservative. ${ }^{4}$ Now, if we restrict our attention to inverses of $B E$ that are increasing and weakly conservative, it turns out that there remain only two.

Lemma 12. Let $\delta$ be an increasing, weakly conservative function from $\wp(D)$ into $\wp(\wp(D))$ such that $\mathrm{BE} \circ \delta$ is the identity map on $\wp(D)$. Then either

(i) $\delta=\mathrm{A}$ or

(ii) $\delta(P)= \begin{cases}\mathrm{A}(P) & \text { if } P \neq D \\ \wp(D) & \text { if } P=D .\end{cases}$

Proof. Let $P \in \wp(D)$ and $x \in P$. By $(8),\{x\} \in$ $\delta(P)$. Since $\delta$ is increasing, for every $Q \in \wp(D)$ such that $\{x\} \subseteq Q$, we have $Q \in \delta(P)$, so lift $(x) \subseteq \delta(P)$. Hence

$$
\mathrm{A}(P)=\bigcup_{x \in P} \operatorname{lift}(x) \subseteq \delta(P) .
$$

We now show that $\delta(P)=\mathrm{A}(P)$ if $P \neq D$. Suppose $\delta(P) \neq \mathrm{A}(P)$. (12) implies $\delta(P) \nsubseteq$ $\mathrm{A}(P)$, so there exists some $Q \in \delta(P)$ such that $Q \notin \mathrm{A}(P)$, which means $P \cap Q=\varnothing$ by the definition of A. Since $\delta$ is weakly conservative and $Q \in \delta(P)$, we have $\varnothing=P \cap Q \in \delta(P)$. Because $\delta$ is increasing, for every $x \in D$, we have $\{x\} \in \delta(P)$ since $\varnothing \in \delta(P)$ and $\varnothing \subseteq\{x\}$. (8) then implies $P=D$.

Finally, observe that by (12),

$$
\begin{aligned}
\delta(D) & \supseteq \mathrm{A}(D) \\
& =\{Q \in \wp(D) \mid D \cap Q \neq \varnothing\} \\
& =\{Q \in \wp(D) \mid Q \neq \varnothing\} \\
& =\wp(D) /\{\varnothing\},
\end{aligned}
$$

so either $\delta(D)=\mathrm{A}(D)=\wp(D) /\{\varnothing\}$ or $\delta(D)=$ $\wp(D)$. It follows that either (i) or (ii) holds.

How can we distinguish $A$ from the other increasing, weakly conservative inverse of BE described in Case (ii) of the above lemma? One possibility might be to note that while $\mathrm{A}(D)$ is a sieve, $\delta(D)$ in Case (ii) is not a sieve, in the sense of Barwise and Cooper (1981):

$$
\begin{aligned}
& \mathscr{P} \in \wp(\wp(D)) \text { is a sieve } \\
& \Leftrightarrow \mathscr{P} \neq \wp(D) \text { and } \mathscr{P} \neq \varnothing .
\end{aligned}
$$

\footnotetext{
${ }^{4}$ Conservativity coincides with Barwise and Cooper's (1981) 'lives-on-its-argument' property, which Barwise and Cooper propose to be a universal property of determiners.
} 
A non-sieve is either true of every predicate or false of every predicate, and therefore would be pointless to use in normal conversation. Van Benthem (1986) suggests that a determiner $\delta$ is generally expected to be such that $\delta(P)$ is a sieve for all $P \neq \varnothing$ (Variety, p. 9).

Another, presumably more appealing way is to invoke the notion of logicality, for which I refer to Westerståhl (1985). So far, we have fixed a model, whose domain of entities is $D$, and have not strictly distinguished linguistic expressions and their model-theoretic interpretations. We should now get rigorous about this distinction because logicality is a property of an object language symbol, and not of its interpretation in a particular model. Henceforth, let's take BE and A to be object language symbols such that for every model $\mathcal{M}=\left\langle D, \llbracket \rrbracket^{\mathcal{M}}\right\rangle$,

$$
\begin{aligned}
\llbracket \mathrm{BE} \rrbracket^{\mathcal{M}} & =\lambda \mathscr{P} .\{x \in D \mid\{x\} \in \mathscr{P}\}, \\
\llbracket \mathrm{A} \rrbracket^{\mathcal{M}} & =\lambda P .\{Q \in \wp(D) \mid P \cap Q \neq \varnothing\} .
\end{aligned}
$$

Now according to Westerståhl (1985), an object language symbol is logical if and only if it has the two properties called constancy and topicneutrality. ${ }^{5}$ It turns out that constancy alone is sufficient to single A out. Here is the relevant definition (Westerståhl, 1985, p. 393, with slight adaptation).

$$
\begin{aligned}
& \text { A determiner } \delta \text { is constant } \\
& \Leftrightarrow \text { for all models } \mathcal{M}_{1}=\left\langle D_{1}, \llbracket \rrbracket^{\mathcal{M}_{1}}\right\rangle \\
& \text { and } \mathcal{M}_{2}=\left\langle D_{2}, \llbracket \llbracket \rrbracket^{\mathcal{M}_{2}}\right\rangle \text {, if } D_{1} \subseteq D_{2}, \\
& \text { then for all } P, Q \subseteq D_{1} \text {, we have } \\
& Q \in \llbracket \delta \rrbracket^{\mathcal{M}_{1}}(P) \text { iff } Q \in \llbracket \delta \rrbracket^{\mathcal{M}_{2}}(P) .
\end{aligned}
$$

A can now be characterized as in the theorem below. So long as we assume all determiners to be conservative, this theorem tells us that $A$ is the only increasing, logical determiner that is an inverse of BE.

Theorem 13. A is the unique increasing, weakly conservative, constant inverse of $\mathrm{BE}{ }^{6}$

Proof. What this theorem asserts precisely is that

\footnotetext{
${ }^{5}$ Constancy corresponds to (invariance for) Extension (of the context) in van Benthem's (1986) terminology. Topicneutrality is a generalized notion of permutation invariance (cf. Keenan and Stavi, 1986; van Benthem, 1986).

${ }^{6}$ Being both conservative and constant is equivalent to being conservative* in Westerståhl's (1985) terminology. So we could alternatively say that $A$ is the unique increasing and conservative* inverse of $\mathrm{BE}$.
}

(i) $\mathrm{A}$ is constant and for every model $\mathcal{M}=$ $\left\langle D, \llbracket \rrbracket^{\mathcal{M}}\right\rangle, \llbracket \mathrm{A} \rrbracket^{\mathcal{M}}$ is increasing and weakly conservative and $\llbracket B E \rrbracket^{\mathcal{M}} \circ \llbracket A \rrbracket^{\mathcal{M}}$ is the identity map on $\wp(D)$,

and that

(ii) if $\delta$ is constant and for every model $\mathcal{M}=$ $\left\langle D, \llbracket \rrbracket^{\mathcal{M}}\right\rangle, \llbracket \delta \rrbracket^{\mathcal{M}}$ is increasing and weakly conservative and $\llbracket \mathrm{BE} \rrbracket^{\mathcal{M}} \circ \llbracket \delta \rrbracket^{\mathcal{M}}$ is the identity map on $\wp(D)$, then for every model $\mathcal{M}$, $\llbracket \delta \rrbracket^{\mathcal{M}}=\llbracket \mathrm{A} \rrbracket^{\mathcal{M}}$.

Showing (i) is straightforward. Here, let's just verify the constancy of $\mathrm{A}$. Let $\mathcal{M}_{1}=$ $\left\langle D_{1}, \llbracket \llbracket \rrbracket^{\mathcal{M}_{1}}\right\rangle$ and $\mathcal{M}_{2}=\left\langle D_{2}, \llbracket \llbracket \rrbracket^{\mathcal{M}_{2}}\right\rangle$ be models such that $D_{1} \subseteq D_{2}$. For all $P, Q \subseteq D_{1}$,

$$
\begin{aligned}
& Q \in \llbracket A \rrbracket^{\mathcal{M}_{1}}(P) \\
\text { iff } & Q \in\left\{R \in \wp\left(D_{1}\right) \mid P \cap R \neq \varnothing\right\} \\
\text { iff } & Q \in\left\{R \in \wp\left(D_{2}\right) \mid P \cap R \neq \varnothing\right\} \\
\text { iff } & Q \in \llbracket A \rrbracket^{\mathcal{M}_{2}}(P) .
\end{aligned}
$$

Thus $\mathrm{A}$ is constant.

To show (ii), assume for a contradiction that $\delta$ has the described properties but there exists some model $\mathcal{M}_{1}=\left\langle D_{1}, \llbracket \llbracket \rrbracket^{\mathcal{M}_{1}}\right\rangle$ such that $\llbracket \delta \rrbracket^{\mathcal{M}_{1}} \neq$ $\llbracket \mathrm{A} \rrbracket^{\mathcal{M}_{1}}$. By Lemma $12, \llbracket \delta \rrbracket^{\mathcal{M}_{1}}\left(D_{1}\right)=\wp\left(D_{1}\right)$, so in particular,

$$
\varnothing \in \llbracket \delta \rrbracket^{\mathcal{M}_{1}}\left(D_{1}\right) .
$$

Now let $\mathcal{M}_{2}=\left\langle D_{2}, \llbracket \rrbracket^{\mathcal{M}_{2}}\right\rangle$ be a model with $D_{2} \supsetneq D_{1}$. By Lemma 12, $\llbracket \delta \rrbracket^{\mathcal{M}_{2}}\left(D_{1}\right)=$ $\llbracket \mathrm{A} \rrbracket^{\mathcal{M}_{2}}\left(D_{1}\right)=\left\{Q \in \wp\left(D_{2}\right) \mid D_{1} \cap Q \neq \varnothing\right\}$, so

$$
\varnothing \notin \llbracket \delta \rrbracket^{\mathcal{M}_{2}}\left(D_{1}\right) .
$$

This contradicts the constancy of $\delta$.

\section{Conclusion}

Given that the domain of entities of a model for natural language semantics should generally be infinite, BE is characterized not as the unique homomorphism that makes the Partee triangle commute (Theorem 8), but as the unique complete homomorphism that makes it commute (Theorem 4). In light of Keenan and Faltz (1985), who have shown the importance of considering complete (rather than plain) homomorphisms in natural language semantics, this is a welcome result. BE can alternatively be characterized as the unique homomorphism that is an inverse of $A$ (Theorem 11), 
while $A$ can be characterized as the unique increasing, (weakly) conservative, constant/logical inverse of $B E$ (Theorem 13). From this viewpoint, the naturalness of $B E$ and that of $A$ complement each other. On the other hand, despite Partee's (1986) conjecture that A and THE are the most "natural" determiners, it is not clear whether THE may be mathematically viewed as equally natural as $A$ is. I hope that this paper has elucidated some finer mathematical points of the Partee triangle that have gone unnoticed and will help rid the linguistic community of any misunderstandings or confusion regarding Partee's (1986) Fact 2. Partee's statement in Fact 2 was not precise, but after all, the results of this paper reinforce her intuition that $\mathrm{BE}$ is nice and natural.

\section{Acknowledgments}

I would like to thank Makoto Kanazawa, who kindly read a very early draft of this paper and informed me that van Benthem (1986), while assuming the domain of entities to be finite, asserted Partee's (1986) Fact 2 without proof.

\section{References}

Jon Barwise and Robin Cooper. 1981. Generalized quantifiers and natural language. Linguistics and Philosophy 4:159-219.

Steven Givant and Paul Halmos. 2009. Introduction to Boolean Algebras. Springer, New York.

Edward L. Keenan and Leonard M. Faltz. 1985. Boolean Semantics for Natural Language. D. Reidel Publishing Company, Dordrecht.

Edward L. Keenan and Jonathan Stavi. 1986. A semantic characterization of natural language determiners. Linguistics and Philosophy 9:253-326.

Gödehard Link. 1983. The logical analysis of plurals and mass terms: A lattice-theoretical approach. In Rainer Bäuerle, Christoph Schwarze, and Arnim von Stechow, editors, Meaning, Use, and Interpretation of Language, Walter de Gruyter, Berlin, pages 303 323.

Barbara H. Partee. 1986. Noun phrase interpretation and type-shifting principles. In Jeroen Groenendijk, Dick de Jongh, and Martin Stokhof, editors, Studies in Discourse Representation Theory and the Theory of Generalized Quantifiers, Foris Publications, Dordrecht, pages 115-143.

Johan van Benthem. 1986. Essays in Logical Semantics. D. Reidel Publishing Company, Dordrecht.
Dag Westerståhl. 1985. Logical constants in quantifier languages. Linguistics and Philosophy 8:387-413. 